\title{
Characterization of myocardial fiber orientation to assess therapeutic exosomes from cardiosphere- derived cells (CDCs) in myocardial infarcted porcine with in vivo diffusion-tensor CMR on a clinical scanner
}

\author{
Christopher T Nguyen ${ }^{1 *}$, James Dawkins², Elizabeth M Tunnicliffe ${ }^{3}$, Xiaoming Bi ${ }^{4}$, Matthew D Robson ${ }^{3}$, Debiao $\mathrm{Li}^{1,5}$, \\ Eduardo Marbán²
}

From 19th Annual SCMR Scientific Sessions

Los Angeles, CA, USA. 27-30 January 2016

\section{Background}

Diffusion-Tensor cardiovascular magnetic resonance (DT-CMR) is capable of mapping myocardial fiber orientation [1,2]. It has been demonstrated in myocardial infarction (MI) murine models that DT-CMR can identify the effects of stem cell therapy on myocardial fiber orientation [3]. However, it remains to be seen if this recent work is translatable to large animal and clinical studies. We propose the application of a well-established in vivo cardiac DT-CMR technique [4] to characterize myocardial fiber orientation before and after the novel regenerative therapy of using intramyocardial injection of exosomes from cardiosphere-derived cells (CDCs) in a MI porcine model.

\section{Methods}

MI was induced in 7 Yucatan mini pigs by balloon occlusion of the mid-LAD for 2.5 hours. The MI was allowed to heal for 4 weeks for all pigs defining baseline. Group $1(\mathrm{~N}=4)$ were treated with exosome proteins derived from CDCs. Group 2 was given a placebo.

CMR was performed at baseline and 4 weeks after treatment on a 3T Siemens Verio with the following: whole-heart 2D multi-slice (WH) morphological CMR, WH function CINE CMR, 3 short axis slice DT-CMR (STEAM, 6 dir, $\mathrm{b}=350 \mathrm{~s} / \mathrm{mm}^{2}, 8 \mathrm{avg}, 2 \mathrm{avg} / \mathrm{BH}$ ), and

${ }^{1}$ Biomedical Imaging Research Institute, Cedars-Sinai Medical Center, Los Angeles, CA, USA

Full list of author information is available at the end of the article
WH viability CMR (LGE PSIR, TI = $315 \mathrm{~ms}$ ). Viability and function CMR yielded scar size (SS) and ejection fraction (EF) [5], respectively. For in vivo DT-CMR, mean diffusivity (MD), fractional anisotropy (FA), and helix angle (HA) maps were calculated. HA transmurality slope (HATS) was calculated by radially sampling the transmural HA along 36 chords and fitting the slope of a linear regression between HA and transmural depth.

Wilcoxon signed-rank test was performed to evaluate the difference between mean slice values $(\mathrm{G} 1 \mathrm{~N}=12$, $\mathrm{G} 2 \mathrm{~N}=9)$ before and after treatment $(\mathrm{p}<0.017$ significance). Change $(\Delta)$ in MD, FA, and HATS were correlated $\left(\mathrm{R}^{\wedge} 2\right)$ with $\triangle \mathrm{SS}$ and $\Delta \mathrm{EF}$.

\section{Results}

For Group 1 (treated), EF, MD, FA, and HATS did not significantly change $\left(\Delta:-1 \pm 2 \%,-0.1 \pm 0.2 \mathrm{um}^{2} / \mathrm{ms}, 0.01 \pm\right.$ 0.03 , and $0.05 \pm 0.15^{\circ} \%$ depth, respectively), while SS was significantly reduced $(\Delta:-3 \pm 2 \%, \mathrm{p}<0.01)$. In contrast, Group 2 (placebo) exhibited significant $(\mathrm{p}<0.01)$ adverse changes with decreased EF $(\Delta:-4 \pm 2 \%)$, increased SS $(\Delta: 3 \pm 1 \%)$, decreased FA $(\Delta:-0.03 \pm 0.02)$, and less helical HATS $\left(-1.2 \pm 0.1\right.$ vs $-0.9 \pm 0.2^{\circ} \%$ depth $) . \Delta$ MD and $\Delta$ FA weakly correlated with $\Delta \mathrm{EF}\left(\mathrm{R}^{\wedge} 2: 0.02\right.$ and 0.27 , respectively) and $\triangle S S\left(R^{\wedge} 2: 0.03\right.$ and 0.08 , respectively). However, $\triangle$ HATS significantly $(\mathrm{p}<0.01)$ correlated highly with $\Delta \mathrm{EF}\left(\mathrm{R}^{\wedge} 2: 0.85\right)$ and $\Delta \mathrm{SS}\left(\mathrm{R}^{\wedge} 2: 0.67\right)$. 


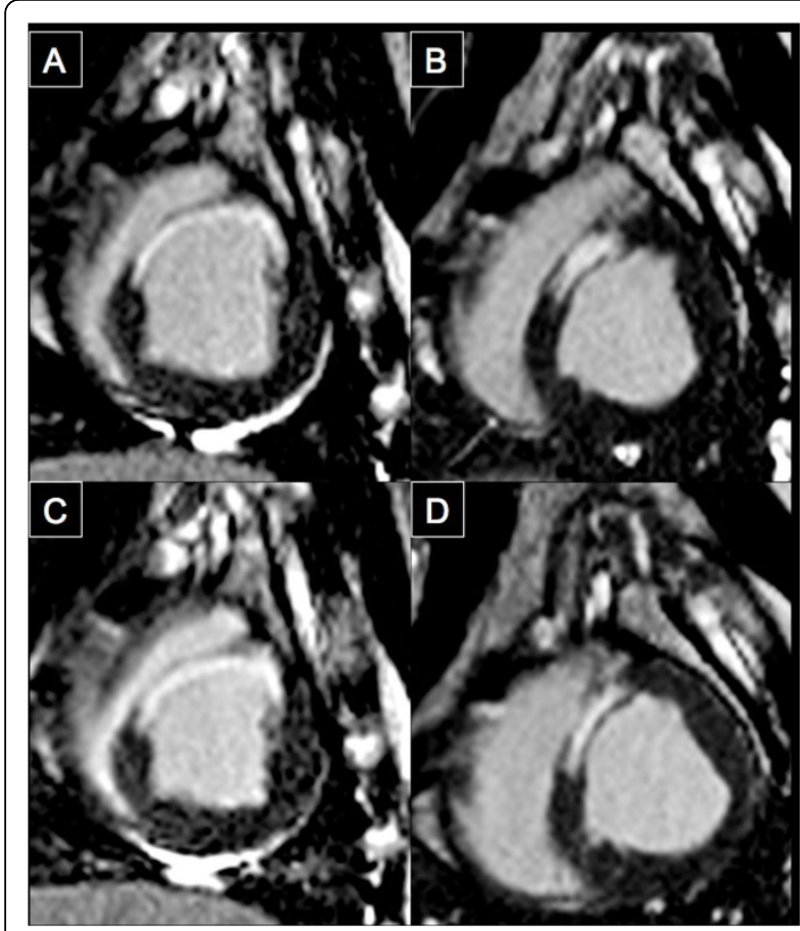

Figure 1 Representative viability images of pre-treatment (A, B) and post-treatment with exosome (C) and placebo (D). Scar size is maintained (1\% difference) with exosome treatment and greatly increased (10\%) with placebo treatment.
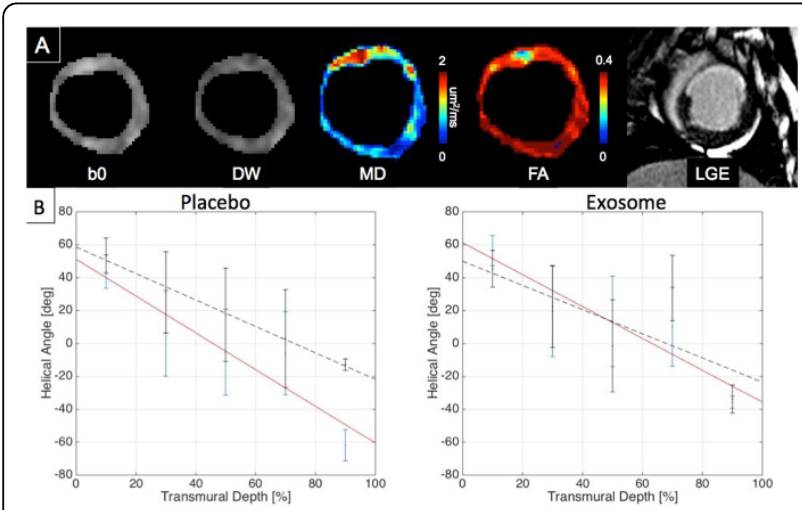

Figure 2 Representative images (A) of least diffusion weighted (b0), diffusion weighted (DW), MD, FA, and LGE of a baseline measurement $\mathbf{4}$ weeks after MI. Scar is demarcated with increases in $M D$, decreases in $F A$, and increase in LGE signal. Representative helix angle vs transmural depth plots (B) before (red line) and after (black dotted line) treatment. Note the placebo treatment causes the slope of the line to flatten out (slope à 0) indicating less helical transmurality, while exosome treatment maintains the helical transmurality.

\section{Conclusions}

In a MI porcine model, in vivo DT-CMR revealed that myocardial fiber orientation was preserved with CDCderived exosome treatment and adversely changed with placebo treatment consistent with observed viability and function changes. Furthermore, changes in helix transmurality highly correlated with changes in viability and function.

\section{Authors' details}

'Biomedical Imaging Research Institute, Cedars-Sinai Medical Center, Los Angeles, CA, USA. ${ }^{2}$ Heart Institute, Cedars-Sinai Medical Center, Los Angeles, CA, USA. ${ }^{3}$ Oxford Centre for Clinical Magnetic Resonance Research, Division of Cardiovascular Medicine, Radcliffe Department of Medicine, University of Oxford, Oxford, United Kingdom. ${ }^{4}$ MR R\&D, Siemens Healthcare, Los Angeles, CA, USA. ${ }^{5}$ Bioengineering, University of California Los Angeles, Los Angeles, CA, USA.

Published: 27 January 2016

\section{References}

1. Reese: MRM 1995.

2. Sosnovik: JCMR 2009.

3. Sosnovik: Circ 2014.

4. Tunnicliffe: JCMR 2014.

5. Malliaras: Circ 2013.

doi:10.1186/1532-429X-18-S1-Q62

Cite this article as: Nguyen et al.: Characterization of myocardial fiber orientation to assess therapeutic exosomes from cardiosphere-derived cells (CDCs) in myocardial infarcted porcine with in vivo diffusion-tensor CMR on a clinical scanner. Journal of Cardiovascular Magnetic Resonance 2016 18(Suppl 1):Q62.

\section{Submit your next manuscript to BioMed Central} and take full advantage of:

- Convenient online submission

- Thorough peer review

- No space constraints or color figure charges

- Immediate publication on acceptance

- Inclusion in PubMed, CAS, Scopus and Google Scholar

- Research which is freely available for redistribution
Biomed Central 